\title{
On the frequency response characteristics of PWM high-speed on-off valves
}

\author{
Yang Shuxing Wang Shulan Lin Xiangzhu \\ Department of Fleight Vehicle Engineering \\ Cao Fan \\ Department of Automatic Control \\ Beijing Institute of Technology \\ P.O.Box 327, Beijing 100081, P. R. China
}

\begin{abstract}
High-speed on-off valves are widely used in PWM electropneumatic servo systems and their characteristics are generallly described by their on and off delay time. This paper focuses on establishing the relationships between their on-off switching behaviors and their frequency response characteristics. A method is proposed by which the frequency response characteristics for an analog PWM high-speed on-off valve at the points that the periods of the input signal are certain times of the carrier ones can be calculated based on its switching behaviors. Then a simple and direct describing function for a PWM high-speed on-off valve is established based on the simulation.
\end{abstract}

\section{KEYWORDS}

PWM, Frequency Response, High-Speed On-Off Valves

\section{NOMENCLATURE}

$$
\begin{aligned}
& \text { Vs }=\text { amplitude of carrier wave } \\
& \varphi_{d}=\text { phase lag }
\end{aligned}
$$

A = amplitude of sine wave

$a_{m}=$ coefficient of Fourier series

\section{INTRODUCTION}

$b_{m}=$ coefficient of Fourier series

$\mathrm{f}_{\mathrm{T}}(\mathrm{t})=$ carrier wave

$\mathrm{T}=$ period of sine wave

$\mathrm{Tc}=$ period of carrier wave

$t_{1}=$ open delay time

$\mathrm{t}_{2}=$ open moving time

$\mathrm{t}_{3}=$ close delay time

$t_{4}=$ close moving time

A high-speed on-off valve is the key element in a PWM fluid power servo system. It has great influence on the control accuracy, the bandwidth and the stability of the system. It is, therefore, necessary to study its static and dynamic behaviors.

Generally, the characteristics of a high-speed on-off valve is described by its on 
and off delay time and the moving time of the valve piston respectively. These four parameters reflect its performances completely and precisely. Especially, they are clear in physical meaning and easy to be obtained by experiments. The static characteristics of a PWM high-speed on-off valve, that is the relationship between the output duty ratio and the input one has, already been widely studied. The influences of its switching behavior and the carrier frequency on the static characteristics have been discovered ${ }^{[1][2]}$. So this paper focuses on the dynamic one.

The frequency domain method is commonly used in the design and the correction of a fluid power servo system. The premise for utilizing this method is that transfer functions or the describing functions of all the elements are known. The describing function of the PWM high-speed on-off valve, therefore, must be discovered in order to use the frequency domain method. As mentioned above, its characteristics is described by the four time domain parameters, and no direct relationship between them can be seen. This paper tries to propose a method by which the relationship between them can be discovered. Based on this method, the influences of the switching behavior of the valve and the carrier frequency on its frequency response characteristics are fully studied. And some conclusions are obtained, which are useful for the designer to identify the high-speed on-off valve correctly during his designing.

A natural sampling analog pulse width modulator with both edges modulated can be divided into three parts, which are an adder, a relay and a carrier wave which is generally a triangular wave ${ }^{[3]}$. Fig 1 shows the construction of a PWM high-speed on-off valve, where $f_{T}(t)$ is the triangular wave with a period $\mathrm{T}_{\mathrm{C}}$ and an amplitude $\mathrm{Vs}$.

The transfer characteristics of the pulse width modulator can be expressed as equation (1).

$$
U=\operatorname{sgn}\left[E+f_{T}(t)\right]
$$

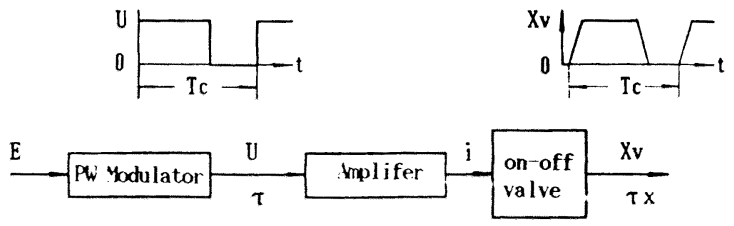

Fig 1. The construction of PWM high-speed on-off valve

\section{CALCULATION METHOD OF THE DESCRIBING FUNCTION}

\section{Assumption}

Working range of the valve: The PWM high-speed on-off vavle works in the linear modulated range in the normal working state. So only the describing function in this range is studied.

If the amplitude of the input sine wave is $A$, the maximum and the minimum duty ratio can be given as follows:

$$
\begin{gathered}
\tau_{\text {max }}=\frac{A}{2 V s}+\frac{1}{2} \\
\tau_{\text {min }}=-\frac{A}{2 V s}+\frac{1}{2}
\end{gathered}
$$

If the high-speed on-off valve works in the linear modulated range, the following inequalitities must be satisfied ${ }^{[2]}$.

$$
\begin{gathered}
\tau_{\max }<1-\left(\tau_{3}+\tau_{4}\right) \\
\tau_{\min }>\tau_{1}+\tau_{2}
\end{gathered}
$$

The amplitude of the input sine wave, therefore, must satisfy the following inequality.

$$
A<V s \cdot \operatorname{Max}\left\{\left[1-2\left(\tau_{1}+\tau_{2}\right)\right],\left[1-2\left(\tau_{3}+\tau_{4}\right)\right]\right\}
$$

The Period of the Input Signal: It is known from equation (1) that $U(t)$ is a periodic signal with a period of $T$ if and only if the ratio between $T$ and the period of the carrier 
wave $\mathrm{Tc}$ is an integer. For the Fourier series method is used in the calculation of the describing function, only those at the points that $\mathrm{U}(\mathrm{t})$ is a periodic function can be calculated.

Switching Behavior of the High-Speed On-Off Valves: It can be easily verified that when $\mathrm{T}=\mathrm{nTc}$ and the inequality (6) are satisfied, there will be $2 n-1$ roots in the interval $[0, T)$ for equation (7).

$$
f_{T}(t)+A \sin \omega t=0
$$

Without loss generality, assume they are $\mathrm{t}_{0}, \mathrm{t}_{1}, \mathrm{t}_{2}, \cdots, \mathrm{t}_{2 \mathrm{n}-2}$. Furthermore, assume $\mathrm{U}(\mathrm{t})$ changes from 0 to $h$ at the points $t_{2 i}$ and return to 0 at the points $t_{2 i+1}$. The width of the positive pulse is, therefore, given by equation (8).

$$
\Delta t_{i}=t_{2 i+1}-t_{2 i}
$$

The piston of the on-off valve cannot follow the pulse strictly and at any time because of its inertia and the delay of the solenoid. Generally, its switching behavior is described by parameters $t_{1}, t_{2}, t_{3}, t_{4}$ as Fig 2 .

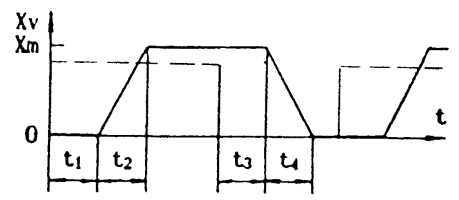

Figure 2. Switching behavior of high-speed on-off valve

During the calculation the assumption that the motion of the piston in the open and close processes is an uniform speed one. The accuracy of the approximation has already been verified by many researches. Thus, the motion of the piston can be given as follows:

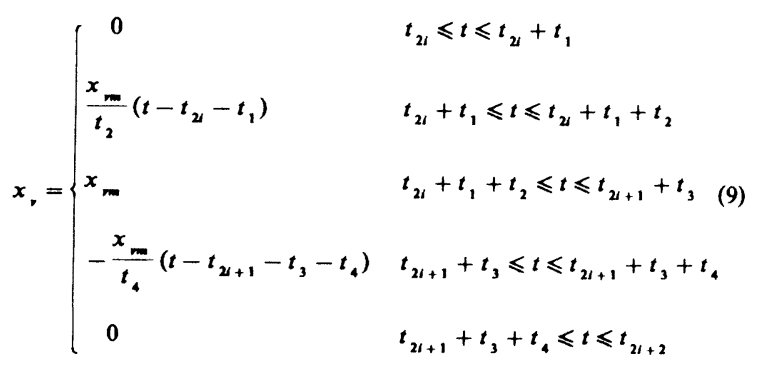

Calculation Method

When a high-speed on-off valve works in PWM mode, its piston must follow $\mathrm{U}(\mathrm{t})$ and work in the synchronized forced oscillation state. If $U(t)$ is a periodic function, $X v$ will also a periodic one with the same period as $U(t)$. $\mathrm{Xv}$, therefore, can be expanded into a Fourier series in the interval $[0, T]$. It will converge to $\mathrm{Xv}$ because the convergent condition is satisfied. The coefficients of the Fourier series are given as follows.

$$
\begin{aligned}
& a_{m}=\frac{2}{T} \int_{0}^{T} x_{v}(t) \cos m \omega t d t \\
& b_{m}=\frac{2}{T} \int_{0}^{T} x_{v}(t) \sin m \omega t d t
\end{aligned}
$$

Both $\mathrm{Xv}(\mathrm{t})$ and $\sin \omega \mathrm{t}$ are with a period of $\mathrm{T}$, therefore, there exists the equation (12).

$$
\int_{0}^{t_{0}} x_{v}(t) \sin m \omega t d t=\int_{T}^{T+t_{0}} x_{v}(t) \sin m \omega t d t
$$

There also exists the equation (13) for the same reason.

$$
\int_{0}^{t_{0}} x_{v}(t) \cos m \omega t d t=\int_{T}^{r+t_{0}} x_{v}(t) \cos m \omega t d t
$$

The expression of $a_{m}$ and $b_{m}$ then can be changed to equation (14) and (15).

$$
\begin{aligned}
& a_{m}=\frac{2}{T} \int_{t_{0}}^{T+t_{0}} x_{v}(t) \cos m \omega t d t \\
& b_{m}=\frac{2}{T} \int_{t_{0}}^{T+t_{0}} x_{v}(t) \sin m \omega t d t
\end{aligned}
$$

The $a_{m}$ and $b_{m}$ can be obtained by substituting the equation (9) into (14) and (15) respectively.

$$
\begin{aligned}
a_{m}= & \frac{2}{T} \sum_{m-1}^{\infty}\left\{\sum_{t=0}^{-1-1} \frac{x_{m}}{t_{2}(m \omega)^{2}}\left[\operatorname{cosm} \omega\left(t_{2}+t_{1}+t_{2}\right)-\operatorname{cosm} \omega\left(t_{21}+t_{1}\right)\right]\right. \\
& \left.-\frac{x_{m}}{t_{4}(m \omega)^{2}}\left[\operatorname{cosm} \omega\left(t_{21+1}+t_{3}+t_{4}\right)-\cos m \omega\left(t_{21+1}+t_{3}\right)\right]\right\}
\end{aligned}
$$




$$
\begin{aligned}
b_{m}= & \frac{2}{T} \sum_{m=1}^{\infty}\left\{\sum_{t=0}^{n-1} \frac{x_{m}}{t_{2}(m \omega)^{2}}\left[\sin m \omega\left(t_{2 l}+t_{1}+t_{2}\right)-\sin m \omega\left(t_{21}+t_{1}\right)\right]\right. \\
& \left.-\frac{x_{m}}{t_{4}(m \omega)^{2}}\left[\sin m \omega\left(t_{2+1}+t_{3}+t_{4}\right)-\sin m \omega\left(t_{2+1}+t_{3}\right)\right]\right\}
\end{aligned}
$$

Because the describing function is determined by $a_{1}$ and $b_{1}$, the amplitued and phase frequency response characteristics can be obtained from the equation (18) and (19).

$$
\begin{gathered}
A_{l}(\omega)=20 \log \frac{\sqrt[2]{a_{1}^{2}+b_{1}^{2}}}{A} \\
\theta_{l}(\omega)=\operatorname{arctg} \frac{a_{1}}{b_{1}}
\end{gathered}
$$

The remaining problem is how to determine the roots $t_{0}, t_{1}, t_{2}, \cdots, t_{2 n-2}$ of equation (7). It is true that there is no way to express them in equations. However, with the help of a computer and by means of the half interval search algorithm it is very simple to find them. The describing function of an analog PWM high-speed on-off valve, thus, can be obtained.

\section{SIMULATION RESEARCH}

The influence of the carrier frequency, the initial phase of the input sine wave and the switching behavior of the high-speed on-off valve on the frequency response characteristics are studied by means of the calculation method.

First, the influence of the initial phase of the input sine wave is studied. During the calculation, the carrier frequency is $100 \mathrm{~Hz}$, the amplitude of the carrier wave Vs is $10 \mathrm{~V}$, the amplitude of the input sine wave $A$ is $4 \mathrm{~V}$ and $\mathrm{t}_{1}=\mathrm{t}_{3}=1 \mathrm{~ms}, \mathrm{t}_{2}=\mathrm{t}_{4}=0.3 \mathrm{~ms}$. Fig 3 illustrates the simulation results when the initial phase are $0^{\circ}, 90^{\circ}, 180^{\circ}$ and $270^{\circ}$ respectively. It can be seen that the initial phase of the input has no influence on both the amplitude bandwidth and the phase lag when $\omega \leqslant \frac{\omega_{c}}{4}$.

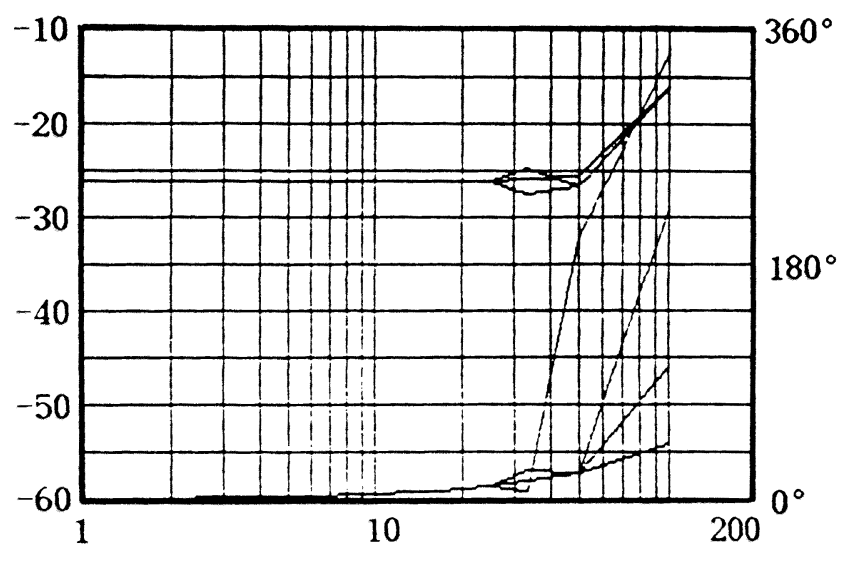

Figure 3. Frequency response characteristic at different initial phase

Secondly, the influence of the carrier frequency is studied.During the calculation, the initial phase of the sine wave is $0^{\circ}$, the amplitude of the carrier wave $\mathrm{Vs}$ is $10 \mathrm{~V}$, the amplitude of the input sine wave $A$ is $4 \mathrm{~V}$ and $\mathrm{t}_{1}=\mathrm{t}_{3}=1 \mathrm{~ms}, \mathrm{t}_{2}=\mathrm{t}_{4}=0.3 \mathrm{~ms}$. Fig 4 shows the simulation results when the carrier frequency is $50 \mathrm{~Hz}, 100 \mathrm{~Hz}$ and $200 \mathrm{~Hz}$ respectively. It can be seen that the bandwidth is $\frac{f_{c}}{4}$, where $f_{c}$ is the carrier frequency.

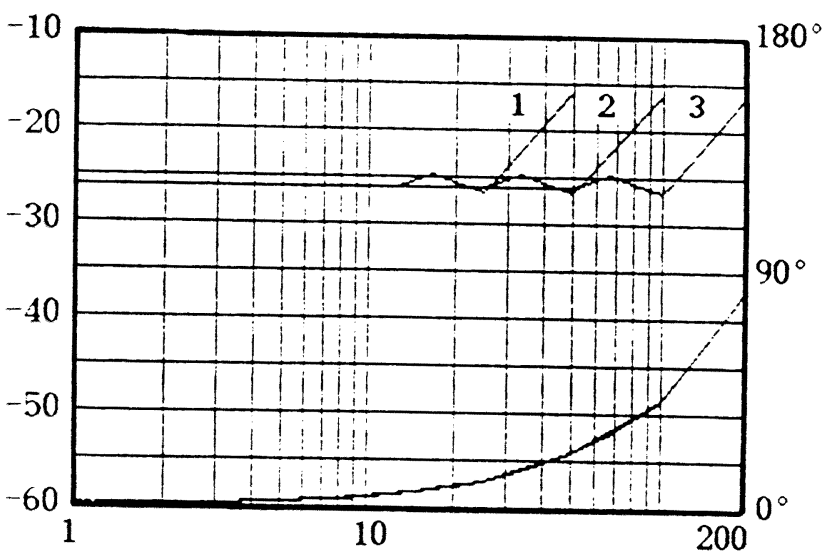

Figure 4. The relationship between bandwidth and carrier frequency

Thirdly, the influence of the switching behavior of the high-speed on-off valve is studied.During the calculation, the carrier frequency is $100 \mathrm{~Hz}$, the amplitude of the carrier wave $\mathrm{Vs}$ is $10 \mathrm{~V}$, the amplitude of the input sine wave $A$ is $4 \mathrm{~V}$ and the initial phase of the input sine wave is $0^{\circ}$. Fig 5 illustrates the 
simulation results with different switching behaviors. In the first curve the four parameters are $t_{1}=t_{3}=1 \mathrm{~ms}, t_{2}=t_{4}=0.3 \mathrm{~m}$. In the second curve $t_{1}=0.4 \mathrm{~ms}, t_{3}=0.5 \mathrm{~ms}$ and $t_{2}=t_{4}=0.3 \mathrm{~m}$. In the third curve $t_{1}=1.5 \mathrm{~ms}, t_{3}=2.0 \mathrm{~ms}$ and $t_{2}=t_{4}=0.3 \mathrm{~m}$. It can be seen that the switching behavior just affect the phase lag and has no influence on the amplitude bandwidth. From large amounts of simulation results it is discovered that the phase lag can be expressed by equation (20) when $\mathrm{T}<4 \mathrm{Tc}$.

$$
\varphi_{d}=\frac{2 t_{1}+2 t_{3}+t_{2}+t_{4}}{4} \omega
$$

\section{CONCLUSIONS}

On the basis of the simulation the following conclusions can be drawn.

1. The bandwidth of a PWM high-speed on-off valve working in the linear modulated range is $\frac{f_{c}}{4}$.
2. The switching behavior of the high-speed on-off valve only affect the phase lag.

3. The describing function of a PWM high-speed on-off valve can be simplified to a pure delay when the period of the input sine wave satisfies $T<4 \mathrm{Tc}$.

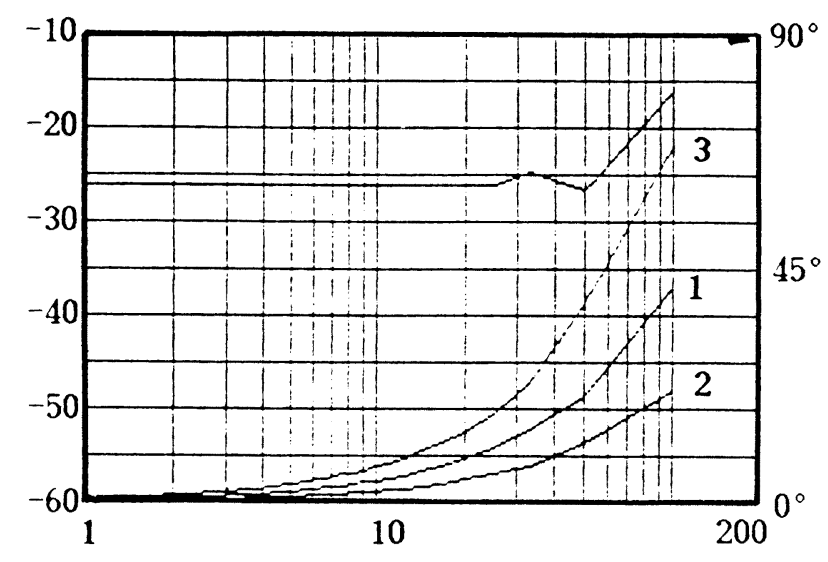

Figure 5. Frequency response with different switching behaviors

\section{REFERENCES}

1. T.Muto, H.Yamada and Y.Suematsu, Digital Simulation of PWM Hydraulic Actuator System, Proc. 2th International Conference On Fluid Power Transmission and Control, Hangzhou, China, March 20-22 1989, pp. 526-531.

2. Yang Shuxing, Wang Shulan Lin Xiangzhu and Cao Fan, The Static Characteristics of Analog PWM High-Speed On-Off Valves, Hydraulics and Pneumatics, 1991, 4, 21-24.

3. Black, H. S., ModulationTheory, D. Van Nostrand Co. Inc., New York, 1953, pp. 263-280. 